\title{
Dietary supplementation with probiotics regulates gut microbiota structure and function in Nile tilapia exposed to aluminum
}

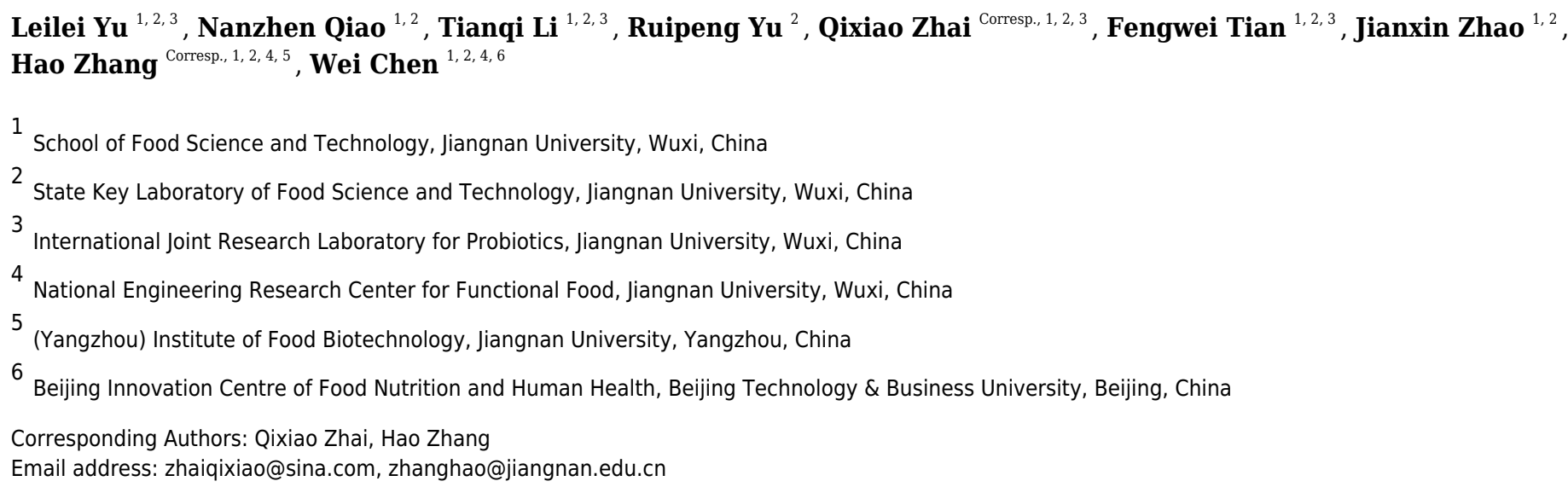

Backgrounds and aims: Aluminum contamination of water is becoming increasingly serious and threatens the health status of fish. Lactobacillus plantarum CCFM639 was previously shown to be a potential probiotic for alleviation aluminum toxicity in Nile tilapia.

Considering the significant role of the gut microbiota on fish health, it seems appropriate to explore the relationships among aluminum exposure, probiotic supplementation, and the gut microbiota in Nile tilapia and to determine whether regulation of the gut microbiota is related to alleviation of aluminum toxicity by a probiotic in Nile tilapia. Methods and results: The tilapia were assigned into four groups, control, CCFM639 only, aluminum only, and aluminum + CCFM639 groups for an experimental period of 4 weeks. The tilapia in the aluminum only group were grown in water with an aluminum ion concentration of 2.73

$\mathrm{mg} / \mathrm{L}$. The final concentration of CCFM639 in the diet was $10^{8} \mathrm{CFU} / \mathrm{g}$. The results show that environmental aluminum exposure reduced the numbers of $L$. plantarum in tilapia feces and altered the gut microbiota. As the predominant bacterial phyla in the gut, the abundances of Bacteroidetes and Proteobacteria in aluminum-exposed fish were significantly elevated and lowered, respectively. At the genus level, fish exposed to aluminum had a significantly lower abundance of Deefgea, Plesiomonas, and Pseudomonas and a greater abundance of Flavobacterium, Enterovibrio, Porphyromonadaceae_uncultured, and Comamonadaceae. When tilapia were exposed to aluminum, the administration of a probiotic promoted aluminum excretion through the feces and led to a decrease in the abundance of Comamonadaceae, Enterovibrio and Porphyromonadaceae. Notably sunplementation with a probiotic only greatly decreased
Peer/ reviewing PDF ( $2018: 11: 32487: 3: 0:$ NEW 4'Abr 2019) 
the abundance of Aeromonas and Pseudomonas. Conclusion: Aluminum exposure altered the diversity of the gut microbiota in Nile tilapia, and probiotic supplementation allowed the recovery of some of the diversity. Therefore, regulation of gut microbiota with a probiotic is a possible mechanism for the alleviation of aluminum toxicity in Nile tilapia. 
1 Dietary supplementation with probiotics regulates gut microbiota structure and function in

2

3 Leilei $\mathrm{Yu}^{1,2,3}$, Nanzhen Qiao ${ }^{1,2}$, Tianqi Li ${ }^{1,2,3}$, Ruipeng $\mathrm{Yu}^{1}$, Qixiao Zhai ${ }^{1,2,3^{*}}$, Fengwei Tian ${ }^{1,2,3}$, 4 Jianxin Zhao $^{1,2}$, Hao Zhang ${ }^{1,2,4,5^{*}}$, and Wei Chen ${ }^{1,2,4,6}$

5

$6{ }^{1}$ State Key Laboratory of Food Science and Technology, Jiangnan University, Wuxi 214122, P.

7 R. China

$8{ }^{2}$ School of Food Science and Technology, Jiangnan University, Wuxi, Jiangsu 214122, China

$9 \quad{ }^{3}$ International Joint Research Laboratory for Probiotics at Jiangnan University, Wuxi, Jiangsu $10 \quad 214122$ China

$11{ }^{4}$ National Engineering Research Center for Functional Food, Jiangnan University, Wuxi, Jiangsu 12 214122, China

135 (Yangzhou) Institute of Food Biotechnology, Jiangnan University, Yangzhou 225004, China

$14{ }^{6}$ Beijing Innovation Centre of Food Nutrition and Human Health, Beijing Technology \& 15 Business University, Beijing 100048, P.R. China

16

17

18

19

20

21

22

*Corresponding author: Dr. Qixiao Zhai.

Tel: 86-510-85912155, Fax: 86-510-85912155

E-mail: zhaiqixiao@sina.com

*Corresponding author: Dr. Hao Zhang.

Tel: 86-510-85912155, Fax: 86-510-85912155

E-mail: zhanghao@jiangnan.edu.cn 


\section{Abstract}

Backgrounds and aims: Aluminum contamination of water is becoming increasingly serious and threatens the health status of fish. Lactobacillus plantarum CCFM639 was previously shown to be a potential probiotic for alleviation aluminum toxicity in Nile tilapia. Considering the significant role of the gut microbiota on fish health, it seems appropriate to explore the relationships among aluminum exposure, probiotic supplementation, and the gut microbiota in Nile tilapia and to determine whether regulation of the gut microbiota is related to alleviation of aluminum toxicity by a probiotic in Nile tilapia.

Methods and results: The tilapia were assigned into four groups, control, CCFM639 only, aluminum only, and aluminum + CCFM639 groups for an experimental period of 4 weeks. The tilapia in the aluminum only group were grown in water with an aluminum ion concentration of $2.73 \mathrm{mg} / \mathrm{L}$. The final concentration of CCFM639 in the diet was $10^{8} \mathrm{CFU} / \mathrm{g}$. The results show that environmental aluminum exposure reduced the numbers of $L$. plantarum in tilapia feces and altered the gut microbiota. As the predominant bacterial phyla in the gut, the abundances of Bacteroidetes and Proteobacteria in aluminum-exposed fish were significantly elevated and lowered, respectively. At the genus level, fish exposed to aluminum had a significantly lower abundance of Deefgea, Plesiomonas, and Pseudomonas and a greater abundance of Flavobacterium, Enterovibrio, Porphyromonadaceae_uncultured, and Comamonadaceae. When tilapia were exposed to aluminum, the administration of a probiotic promoted aluminum excretion through the feces and led to a decrease in the abundance of Comamonadaceae, Enterovibrio and Porphyromonadaceae. Notably, supplementation with a probiotic only greatly decreased the abundance of Aeromonas and Pseudomonas.

Conclusion: Aluminum exposure altered the diversity of the gut microbiota in Nile tilapia, and probiotic supplementation allowed the recovery of some of the diversity. Therefore, regulation of gut microbiota with a probiotic is a possible mechanism for the alleviation of aluminum toxicity in Nile tilapia. 


\section{Introduction}

Aluminum, the third commonest chemical element and the most abundant metal on earth, is ubiquitous in the environment. In recent years, environmental aluminum levels have increased due to diverse anthropogenic activities such as water treatment, eutrophic lakes control, mining operations, and industrial landfill (Fernandez-Davila et al. 2012; Garcia-Medina et al. 2011).The aluminum level reaches $5.7 \mathrm{mg} / \mathrm{L}$ in certain rivers and lakes in England, the United States, China, and Brazil (Camargo et al. 2009; da Cruz et al. 2015), research has been shown that concentration of aluminum ranging from 0.1 to $0.2 \mathrm{mg} / \mathrm{L}$ can be harmful to fish (Baker \& Schofield 1982; Wang et al. 2013). Excessive aluminum can accumulate in multiple fish tissues and organs and exert adverse effects on the blood circulation and on endocrine, metabolic and reproductive function (Azmat et al. 2012). Excess aluminum ions in water were reported to cause mortalities and decreasing population of Atlantic salmon in Norway, southeast Canada, and the northeastern United States (Monette \& McCormick 2008). Aluminum contamination causes economic losses in aquaculture and poses potential human health risks from consumption of aquatic products.

Microorganisms produce a variety of metabolites that can have remarkable effects on the external environment and on the host, including changes in $\mathrm{pH}$, suppression of inflammation, and detoxification (Berdy 2005; Louis et al. 2014). Hence, the gut microbiota can significantly alter the host's physiology and its metabolism of nutrients and exogenous toxic substances, and it can shape the microbiome and immune systems (Ni et al. 2014). They may be important mediators of the bioavailability and toxicity of toxic metals. Indeed, long-term toxic metal exposure, including aluminum, lead and chromium, altered the composition of intestinal microbiota (Wu et al. 2017; Zhai et al. 2017).

Lactic acid bacteria probiotics, which are generally derived from humans or food products, are generally recognized as safe (GRAS) strains (Farnworth 2008) and have been widely applied in various situations, including aquaculture, to improve food safety (Sihag \& Sharma 2012). Some probiotics have been used in the culture of some aquatic organisms to promote growth and control infectious disease. They can improve the host's intestinal microflora balance and increase the protective effect against pathogenic bacteria (Lahti et al. 2013; Pirarat et al. 2015). For example, L. johnsonii La1, L. rhamnosus LC705, B. lactis Bb12, L. casei Shirota and others can effectively control infection with Vibrio anguillarum, Flavobacterium psychrophilum and Aeromonas salmonicida, to prevent furunculosis in rainbow trout (Nikoskelainen et al. 2001), and L. rhamnosus GG can control infection of tilapia by Edwardsiella tarda and Streptococcus agalactiae (Pirarat et al. 2006; Pirarat et al. 2015). 
Our previous study showed that supplementation with probiotic L. plantarum CCFM639, a strain with superb aluminum binding and tolerance abilities, decreased the aluminum level in tissues and alleviated aluminum toxicity by preventing oxidative stress and histopathological changes in tilapia ( $\mathrm{Yu}$ et al. 2017). However, it remains unclear whether the mechanisms of recovery and alleviation are related to the gut microbiota. Therefore, we investigated alterations in the composition and structure of the intestinal microbiota in tilapia after aluminum exposure and the addition $L$. plantarum CCFM639 to their daily feed.

Tilapia is one of the most important aquatic species in aquaculture worldwide and is farmed in more than 120 countries and territories (Junning et al. 2018). In 2015, its production accounted for more than $10 \%$ of all farmed fish worldwide (FAO 2015). Moreover, tilapia are recognized as a good biological model because they are easy to handle, culture, and maintain in the laboratory (Korkmaz et al. 2009), and because they display excellent stress sensitivity (Zheng et al. 2016). The aim of the study was to explore whether the regulation of gut microbiota is an aluminum toxicity alleviation mechanism exerted by probiotics in tilapia.

\section{Materials and methods}

\subsection{Probiotic and fish diet preparation}

L. plantarum CCFM639, kindly provided by the in-house Culture Collections of Food Microbiology (CCFM), Jiangnan University (Wuxi, China), was inoculated in MRS broth (Qingdao Hopebio, China) and in a static condition at $37{ }^{\circ} \mathrm{C}$ for $18 \mathrm{~h}$. After centrifugation at $8000 \mathrm{~g}$ at $4^{\circ} \mathrm{C}$ for $5 \mathrm{~min}$, the medium was removed and the cell pellets were suspended with sterile normal saline solution $(0.85 \%)$ to one-hundredth of the original medium volume. The pellets were mixed with the fish basal diet using a sterile spreader to distribute the bacterial cells evenly and to achieve a final probiotic concentration of $10^{8} \mathrm{CFU} / \mathrm{g}$ in the feed. The formula and nutrient levels of the basal fish diet were consistent with those in previous studies ( $\mathrm{Yu}$ et al. 2017). The dose of the L. plantarum strain was selected on the basis of previous reports (Heo et al. 2013; Ridha \& Azad 2012; Ridha \& Azad 2016). The bacterial concentration in the fish diet was also confirmed by colony counting. The bacteria-containing feed was prepared weekly and stored at $4^{\circ} \mathrm{C}$ before use.

\subsection{Experimental design}

One hundred ninety-two male tilapia were purchased from the Freshwater Fisheries Research Centre of the Chinese Academy of Fishery Sciences in Wuxi and stocked in a cylindrical aquarium $\left(0.6 \mathrm{~m}^{2} \times 0.85 \mathrm{~m}\right)$ at a fish loading ratio of $1.09 \mathrm{~g} / \mathrm{L}$ for 3 weeks. The average $( \pm \mathrm{SEM})$ weight of the tilapia was $34.01 \pm 0.19 \mathrm{~g}$. The amount of feed consumed each day was $3 \%$ of the average body weight of the fish. The fish were fed manually at 9 am and $5 \mathrm{pm}$ 
each day. After a 3-week adaptation period, the fish were fasted for 1 day. The four groups are listed in Table 1, including the control group, the probiotic group (639 only), the aluminum exposure group ( $\mathrm{Al}$ only) and the probiotic intervention group $(\mathrm{Al}+639)$ randomly. The fish in each group were randomly assigned to three tanks with 16 tilapias in each tank. In the aluminum exposure group, the tilapia were grown in water with an aluminum ion concentration $\left(\mathrm{AlCl}_{3} \cdot 6 \mathrm{H}_{2} \mathrm{O}\right)$ of $2.73 \mathrm{mg} / \mathrm{L}$. The selection of this dose was based mainly on the aluminum exposure doses reported in drinking water, rivers and lakes in previous studies (Yu et al. 2017). The test period was 4 weeks, the freshwater was changed and the aluminum level in the water was checked every 2 days. Inductively coupled plasma mass spectrometry (ICP-MS; NexIon300X, PerkinElmer) was used to determine the amount of aluminum in the water.

To ensure that fecal samples were not affected by the high level of waterborne aluminum, they were collected quickly after the water was changed to normal water (without aluminum). After collection of the fecal samples, the aluminum ions were added into the water. At the end of the assay, the tilapia were sacrificed under anesthesia after $24 \mathrm{~h}$ of fasting. The entire intestinal tract was removed under aseptic conditions and $0.2 \mathrm{~g}$ of intestinal contents was squeezed and collected in sterile tubes for analysis of the intestinal microbiota.

The animal experiments was approved by the Ethics Committee of Jiangnan Universi ty, China (JN No. 20151027-1129-3), and all procedures about the care and use of exper imental animals followed the guidelines set by the European Community (directive 2010/ $63 / \mathrm{EU})$.

\subsection{Determination of fecal aluminum levels}

One gram sample of feces was transferred to a microwave digestion tank (OMNI; CEM, UK) with $70 \%$ concentrated nitric acid. The microwave digestion system (MARS; CEM, UK) was used. The heating procedure of digestion included three stages: stage 1 - power $2000 \mathrm{~W}$, ramp 3:00, temperature $120^{\circ} \mathrm{C}$, hold 3:00; stage 2-power $2000 \mathrm{~W}$, ramp 3:00, temperature $150^{\circ} \mathrm{C}$, hold 10:00; stage 3-power $2000 \mathrm{~W}$, ramp 5:00, temperature $190^{\circ} \mathrm{C}$, hold 16:00. After the temperature fell below $50^{\circ} \mathrm{C}$, the samples were removed and diluted to $50 \mathrm{~mL}$ with deionized water. ICP-MS (NexIon-300X, PerkinElmer) was used to determine the amount of aluminum in the samples (Ciavardelli et al. 2012).

\subsection{RT-qPCR analyses for $m R N A$ expression of $L$. plantarum in feces}

The $0.2 \mathrm{~g}$ of fecal samples were collected to extract the total genomic DNA following the instructions of the FastDNA Spin Kit (MP Biomedicals, California,). The fecal genomic DNA was used as a template, and real-time quantitative polymerase chain reaction (RT-qPCR; CFX96, 
Bio-Rad) was carried out using the specific primer. The primer sequences $\left(5^{\prime}-3^{\prime}\right)$ referred to previous study (Wang 2018) and as follows, LP-F GGAGCCGCTATTAGTATTTTCAT and LP-R AATACAAGCAAGTCTT185GGACCAG. The $\mathrm{Ct}$ value of the fecal sample fluorescence quantification was brought into the corresponding standard curve to calculate the copy number, which was converted into the amount per gram of feces. The standard curve of RT-qPCR was as follows: $\mathrm{Ct}=-3.1434 * \lg$ (copies) $+36.977\left(\mathrm{R}^{2}=0.9913\right)$ (Wang 2018).

\subsection{Analysis of gut microbiota}

DNA was extracted from $0.2 \mathrm{~g}$ of the contents of the whole intestinal tract using an EZNA DNA Kit (Omega Bio-tek, Georgia, USA) and stored at $-20^{\circ} \mathrm{C}$. PCR amplification of the $16 \mathrm{~S}$ rRNA gene was conducted using the forward primer 515F (5'-barcodeGTGCCAGCMGCCGCGG-3') and the reverse primer 907R (5'CCGTCAATTCMTTTRAGTTT-3') (Zhai et al. 2016). Different samples were distinguished with an 8-base barcode and sequenced using a miseq sequencer (Illumina, Inc., California, USA; illumina miseq PE250). The raw data were quality-filtered and aligned using Trimmomatic and FLASH. The operational taxonomic units (OTUs) were clustered with a 97\% similarity cutoff using UPARSE (http://drive5.com/uparse/). The chimeric sequences were identified and removed using UCHIME (http://www.drive5.com/uchime/). The OTU germline type was identified by the RDP classifier (http://rdp.cme.msu.edu/) against the SILVA (SSU115) 16S rRNA database using a confidence threshold of 70\% (Gajardo et al. 2016; Huyben et al. 2018).

\subsection{Data analysis}

The experimental results were analyzed and tested using analysis of variance and nonparametric tests. The alpha diversity (Chao and Shannon indices) and the beta diversity (PERMANOVA) of the microbiome were calculated based on the OTU level. Tukey's post hoc test of one-way analysis of variance (ANOVA) were performed in aluminum and L. plantarum levels of feces. A P value of less than 0.05 was used as a cutoff to indicate a statistically significant difference. The results were plotted using Origin 8.6 software (Originlab, Massachusetts, USA).

\section{Results}

\subsection{Aluminum level in feces}

Table 2 (DatasetS1) shows the weekly changes in the fecal aluminum content in tilapia. The fecal aluminum content was very low in the control and CCFM639 only groups, and was markedly elevated after aluminum exposure $(\mathrm{P}<0.05)$. Moreover, the aluminum level in feces 
also increased as the duration of aluminum exposure increased. CCFM639 treatment promoted the elimination of aluminum in the feces. At the fourth week, the fecal aluminum level in the aluminum only group was $25.67 \mathrm{mg} / \mathrm{kg}$. With the continuous administration of CCFM639, the fecal aluminum levels were significantly greater than those in the Al-only group at each test point $(\mathrm{P}<0.05)$, up to $35.46 \mathrm{mg} / \mathrm{kg}$ at the third week.

\subsection{Quantification of Lactobacillus in feces}

Table 3 (DatasetS2) shows that the amount of L. plantarum in tilapia feces in the CCFM639only group was higher than control group by two orders of magnitude. By week 4 , the content of L. plantarum in tilapia feces had been significantly decreased by aluminum exposure, from $10^{5.4}$ copies per gram of feces to $10^{5.0}$ copies per gram of feces $(\mathrm{P}<0.05)$, whereas the addition of CCFM639 led to a significant increase in the L. plantarum content in feces to that in the 639only group.

\subsection{Intestinal microbial diversity and composition}

The numbers of OUTs of each group were 147.3, 172.0, 243.7, 206.3, respectively. Concerning alpha diversity microbiome analysis, Shannon and Chaol indices were found higher in the 639 only and the $\mathrm{Al}+639$ treatment groups compared to the control (Figure 1, DatasetS3). As shown in Figure 2A (DatasetS3), Principal Coordinate Analysis based on Unweighted unifrac distance indicated a overall significant clustering on the fecal microbiota composition $(\mathrm{P}=0.002)$, in which PC1 explained $65.75 \%$ of the difference. Moreover, the results of PC1 analysis show that aluminum treatment had the greatest effect on the composition of the gut microbiota in tilapia (Figure 2B, DatasetS3, $\mathrm{P}<0.05$ ).

Aluminum exposure changed the composition of the gut microbiota in Nile tilapia. The four predominant bacterial phyla Fusobacteria, Proteobacteria, Bacteroidetes, and Firmicutes, accounted for $53.74 \%, 38.21 \%, 7.54 \%$, and $0.38 \%$, respectively, in control group (Figure 3, DatasetS4). Fish exposed to aluminum had a significantly greater abundance of Bacteroidetes and fewer Firmicutes, whereas administration of CCFM639 had the opposite effect. Interestingly, CCFM639 administration led to an increase in the abundance of Fusobacteria and Firmicutes and a decrease in the abundance of Proteobacteria.

As shown in Figure 4 (DatasetS5), 17 dominant genera were detected, the five most dominant were Cetobacterium, Deefgea, Plesiomonas, Flavobacterium and Cytophagales. Aluminum exposure significantly reduced the abundance of Plesiomonas, Deefgea, and Pseudomonas and drastically increased the abundance of Flavobacterium, Enterovibrio, and the 
214

215

216

217

218

219

220

221

222

families Porphyromonadaceae and Comamonadaceae (Figure 5 and Table 4, DatasetS5; P<0.05). In the tilapia exposed to aluminum, the administration of L. plantarum CCFM639 further led to a decrease in the abundance of Enterovibrio, Comamonadaceae and Porphyromonadaceae $(\mathrm{P}<0.05)$. Unlike the results in the control group, supplementation with L. plantarum CCFM639 greatly reduced the abundance of Aeromonas and Pseudomonas $(\mathrm{P}<0.05)$.

\section{Discussion}

Aquatic animals, especially fish, have direct contact with the water environment, which contains various pollutants and ever-changing microbiota (Egerton et al. 2018). An excessive aluminum concentration in the aquatic ecosystem can be harmful to the physiological functions of fish, and even threaten their survival (Egerton et al. 2018). In addition, the gut microbiota, which has a close association with fish health, can affect its metabolism, physiology, and immune function, and great inter-individual microbial diversity exists (Sullam et al. 2012). In this study, we adopted a culture-independent technology, next-generation sequencing, which is an up-to-date analytical method that can be widely used for analysis of host microbiota, including that of fish. More specifically, next-generation sequencing can be used to provide detailed information of low abundance microbiota can be provided and the genetic potential of species can even be predicted (Ghanbari et al. 2015). Our results show that the dominant four phyla in Nile tilapia were Fusobacteria, Proteobacteria, Bacteroidetes, and Firmicutes. Proteobacteria, Bacteroidetes, and Firmicutes accounted for $90 \%$ of the gut microbiota in a previous study of the teleost fishes (Giatsis et al. 2015; Liu et al. 2013; Ran et al. 2015). The intestinal microbiota of fish species including tilapia is characterized by various levels of Actinobacteria, Bacteroidetes, Fusobacteria, Proteobacteria and Firmicutes (Adeoye et al. 2016; Wu et al. 2013). At the genus level, the relative abundance of dominant Cetobacterium (affiliated with Fusobacteria) and Plesiomonas were also consistent with the results of a previous study in zebrafish (Ma et al. 2018).

Despite various studies focusing on aluminum exposure and its effects on various host organs, including the liver, kidneys, and brain (Park et al. 2015), the link between aluminum exposure and the host intestinal microbiota remains unclear. The fish gut microbiota is commonly treated as an organ with a significant role in essential physiological functions and overall health. Consistent with our hypothesis, the fecal aluminum level of tilapia increased significantly when the fish were exposed to aluminum, and CCFM639 supplementation promoted aluminum excretion in feces as its excellent aluminum binding ability (Table 1, Figure 6). These results are consistent with our previous study in a mouse model ( $\mathrm{Yu}$ et al. 2016). In addition, the amount of L. plantarum in the tilapia feces increased markedly after the fish 
ingested feed mixed with a probiotic (Table 2). It is notable that aluminum exposure reduced the numbers of $L$. plantarum in tilapia feces at week 4 (Table 3 ), possibly due to a decrease in aluminum-intolerant $L$. plantarum in the gut caused by aluminum exposure.

Environmental exposure to aluminum altered the structure and relative abundance of the intestinal microbiota of Nile tilapia, resulting in a significant decrease in the relative abundance of the phyla Firmicutes and Proteobacteria and the genera Deefgea, Plesiomonas and Pseudomonas and elevated the relative abundance of the phylum Bacteroidetes, and the genera Comamonadaceae and Porphyromonadaceae, Flavobacterium and Enterovibrio $(\mathrm{P}<0.05$; Figures 3, 4, and 5). The alpha diversity results show no significant difference between the control and 639 groups, and between the $\mathrm{Al}$ only and $\mathrm{Al}+639$ group. Accroding to previous studies (Qin et al. 2018; Uronis et al. 2011), we hypothesized that administration of a single probiotic CCFM639 may not exert a dramatic effect on the richness of the whole gut microbiota, but it may induce alterations in the abundance of specific genera. A previous study demonstrated that toxic metal exposure can influence the gut microbiota composition in mice (Zhai et al. 2017). Ingestion of aluminum damage the intestinal mucosa and reduce the intestinal barrier function and immune function (Vignal et al. 2016). Pathogens and opportunistic pathogens in the intestinal tract can take the opportunity to multiply, causing disordered gut microbiota (Berg 1996). A lower relative abundance of phylum Proteobacteria and a higher relative abundance of genus Cetobacterium were found after aluminum exposure. Exposure to silver led to a similar alteration in male zebrafish (Ma et al. 2018). The pathogen Flavobacterium is considered responsible for several fish diseases, including fry syndrome and bacterial cold water disease, which can cause high mortality levels in young fish (Leal et al. 2010; Nematollahi et al. 2003). The increase in the relative abundance of Flavobacterium may be due to its good aluminum tolerance capacity (more than 2000 ppm) (Konishi et al. 1994). Similar speculation can be used to explain the increase in the relative abundance of Comamonadaceae. A series of metal-resistant genes and gene clusters was found in the whole-genome sequencing of the Comamonas strain, including arsenic, stibium, copper and so forth (Li et al. 2013; Lin et al. 2015). Wu et al. studied the virulence of several Enterovibrio and Vibrio strains for zebrafish; the results showed that Enterovibrio had a high level of virulence $\left(\mathrm{LD}_{50}\right.$ values around $\left.10^{4} \mathrm{CFU} / \mathrm{g}\right)$, and that Vibrio had a moderate level of virulence $\left(\mathrm{LD}_{50}\right.$ values around $10^{6} \mathrm{CFU} / \mathrm{g}$ ) (Wu et al. 2015). Enterovibrio promotes the production of indole, which is a toxin that is harmful to intestinal lactic acid bacteria in excess quantities (Nowak \& Libudzisz 2006; Pascual et al. 2009). Porphyromonadaceae is a family in the of Bacteroidetes phylum that can also exert negative effects on the host (Russell et al. 2015). Therefore, aluminum exposure led to an increase in the relative abundance of some harmful bacteria. The dynamic changes in the gut microbiota can 
283

284

directly affect the intestinal mucosa and indirectly affect the health of the fish (Perez et al. 2010). The results help to further explain the harmful effects of aluminum exposure on the host's growth and antioxidant system in previous study (Yu et al. 2017).

Probiotics have shown an excellent ability to resist disease and prevent pathogens (Hai 2015). Probiotic supplementation results in improvements in microvilli density and length, which can increase the absorptive surface of the fish intestines and ultimately enhance the host's physical barrier against potential pathogens (Standen et al. 2016). The use of probiotics stimulates the proliferation of a few probiotic bacteria and decreases the potential pathogens in fish. Numerous studies have demonstrated the significant effects of probiotics in protecting aquatic animals against infection by pathogens, such as the effects of Bacillus spp. against Streptococcus iniae (Cha et al. 2013), and the effects of Pseudomonas spp. against $F$. psychrophilum (Korkea-aho et al. 2012). Compared with the Al-only group, the administration of CCFM639 significantly reduced the abundance of Enterovibrio, Comamonadaceae and Porphyromonadaceae, indicating the potential protective effect of CCFM639 treatment against aluminum-induced increases in pathogen. Aeromonas is one of the fish pathogens that causes several fish diseases, including motile aeromonad septicemia, furunculosis, ulcer disease, and carp erythrodermatitis (Miller \& Harbottle 2018). In 639 only group, the abundance of Aeromonas.was decreased, which indicates modification of the bacterial community composition by administration of the probiotic CCFM639. Studies in tilapia have also demonstrated an improvement in gut microbiota with the addition of probiotics (Standen et al. 2015).

\section{Conclusions}

In conclusion, the accumulated aluminum in Nile tilapia can be excreted through feces, and oral administration of $L$. plantarum CCFM639 increased aluminum excretion (Figure 6). Moreover, environmental exposure to aluminum altered the composition of the gut microbiota, and alteration in the levels of Enterovibrio, Comamonadaceae, and Porphyromonadaceae can be recovered by administration of CCFM639. Therefore, this study provides a further explanation of the protective mechanisms of CCFM639 against aluminum toxicity. Apart from promotion of aluminum discharge through feces, regulation of the gut microbiota with L. plantarum CCFM639 may be an underlying mechanism by which aluminum toxicity is alleviated.

\section{Reference}

Adeoye AA, Jaramillo-Torres A, Fox SW, Merrifield DL, and Davies SJ. 2016. Supplementation of formulated diets for tilapia (Oreochromis niloticus) with selected exogenous enzymes: Overall 
performance and effects on intestinal histology and microbiota. Animal Feed Science and Technology 215:133-143.

Azmat H, Javed M, and Jabeen G. 2012. Acute Toxicity of Aluminium to the Fish (Catla catla, Labeo rohita and Cirrhina mrigala). Pakistan Veterinary Journal 32:85-87.

Baker JP, and Schofield CL. 1982. Aluminum toxicity to fish in acidic waters. Long-Range Transport of Airborne Pollutants: Springer, 289-309.

Berdy J. 2005. Bioactive microbial metabolites. J Antibiot (Tokyo) 58:1-26.

Berg RD. 1996. The indigenous gastrointestinal microflora. Trends in Microbiology 4:430-435.

Camargo MMP, Fernandes MN, and Martinez CBR. 2009. How aluminium exposure promotes osmoregulatory disturbances in the neotropical freshwater fish Prochilus lineatus. Aquatic Toxicology 94:40-46.

Cha JH, Rahimnejad S, Yang SY, Kim KW, and Lee KJ. 2013. Evaluations of Bacillus spp. as dietary additives on growth performance, innate immunity and disease resistance of olive flounder (Paralichthys olivaceus) against Streptococcus iniae and as water additives. Aquaculture 402:50-57.

Ciavardelli D, Consalvo A, Caldaralo V, Di Vacri ML, Nisi S, Corona C, Frazzini V, Sacchetta P, Urbani A, Di Ilio C, and Sensi SL. 2012. Characterisation of element profile changes induced by long-term dietary supplementation of zinc in the brain and cerebellum of 3xTg-AD mice by alternated cool and normal plasma ICP-MS. Metallomics 4:1321-1332.

da Cruz AL, Prado TM, da Silva Maciel LA, and Couto RD. 2015. Environmental effects on the gills and blood of Oreochromis niloticus exposed to rivers of Bahia, Brazil. Ecotoxicology and Environmental Safety 111:23-31.

Egerton S, Culloty S, Whooley J, Stanton C, and Ross RP. 2018. The Gut Microbiota of Marine Fish. Frontiers in Microbiology 9.

FAO. 2015. Global Aquaculture Production.

Farnworth ER. 2008. The evidence to support health claims for probiotics. The Journal of nutrition 138:1250S-1254S.

Fernandez-Davila ML, Razo-Estrada AC, Garcia-Medina S, Gomez-Olivan LM, Pinon-Lopez MJ, Ibarra RG, and Galar-Martinez M. 2012. Aluminum-induced oxidative stress and neurotoxicity in grass carp (Cyprinidae--Ctenopharingodon idella). Ecotoxicol Environ Saf 76:87-92.

Gajardo K, Rodiles A, Kortner TM, Krogdahl Å, Bakke AM, Merrifield DL, and Sørum H. 2016. A highresolution map of the gut microbiota in Atlantic salmon (Salmo salar): A basis for comparative gut microbial research. Scientific Reports 6:30893.

Garcia-Medina S, Razo-Estrada C, Galar-Martinez M, Cortez-Barberena E, Gomez-Olivan LM, AlvarezGonzalez I, and Madrigal-Bujaidar E. 2011. Genotoxic and cytotoxic effects induced by aluminum in the lymphocytes of the common carp (Cyprinus carpio). Comp Biochem Physiol C Toxicol Pharmacol 153:113-118. 
Ghanbari M, Kneifel W, and Domig KJ. 2015. A new view of the fish gut microbiome: Advances from nextgeneration sequencing. Aquaculture 448:464-475.

Giatsis C, Sipkema D, Smidt H, Heilig H, Benvenuti G, Verreth J, and Verdegem M. 2015. The impact of rearing environment on the development of gut microbiota in tilapia larvae. Scientific Reports 5:18206.

Hai N. 2015. The use of probiotics in aquaculture. Journal of applied microbiology 119:917-935.

Heo W-S, Kim Y-R, Kim E-Y, Bai SC, and Kong I-S. 2013. Effects of dietary probiotic, Lactococcus lactis subsp. lactis I2, supplementation on the growth and immune response of olive flounder (Paralichthys olivaceus). Aquaculture 376-379:20-24.

Huyben D, Sun L, Moccia R, Kiessling A, Dicksved J, and Lundh T. 2018. Dietary live yeast and increased water temperature influence the gut microbiota of rainbow trout. Journal of applied microbiology 124:1377-1392.

Junning C, PingSun L, Yongju L, Xinhua Y, and Yongming Y. 2018. Improving the performance of tilapia farming under climate variation. FAO FISHERIES AND AQUACULTURE TECHNICAL PAPER 608.

Konishi S, Souta I, Takahashi J, Ohmoto M, and Kaneko S. 1994. Isolation and Characteristics of AcidTolerant and Aluminum-Tolerant Bacterium. Bioscience Biotechnology and Biochemistry 58:19601963.

Korkea-aho TL, Papadopoulou A, Heikkinen J, von Wright A, Adams A, Austin B, and Thompson KD. 2012. Pseudomonas M162 confers protection against rainbow trout fry syndrome by stimulating immunity. Journal of applied microbiology 113:24-35.

Korkmaz N, Cengiz EI, Unlu E, Uysal E, and Yanar M. 2009. Cypermethrin-induced histopathological and biochemical changes in Nile tilapia (Oreochromis niloticus), and the protective and recuperative effect of ascorbic acid. Environ Toxicol Pharmacol 28:198-205.

Lahti L, Salonen A, Kekkonen RA, Salojarvi J, Jalanka-Tuovinen J, Palva A, Oresic M, and de Vos wM. 2013. Associations between the human intestinal microbiota, Lactobacillus rhamnosus GG and serum lipids indicated by integrated analysis of high-throughput profiling data. PeerJ 1.

Leal CAG, Carvalho-Castro GA, Sacchetin PSC, Lopes CO, Moraes AM, and Figueiredo HCP. 2010. Oral and parenteral vaccines against Flavobacterium columnare: evaluation of humoral immune response by ELISA and in vivo efficiency in Nile tilapia (Oreochromis niloticus). Aquaculture International 18:657-666.

Li J, Wang Q, Zhang S, Qin D, and Wang G. 2013. Phylogenetic and genome analyses of antimony-oxidizing bacteria isolated from antimony mined soil. International Biodeterioration \& Biodegradation 76:7680.

Lin L, Zhu W, Zhan C, Xu B, Wang G, and Luo M. 2015. High correlation between genotypes and phenotypes of environmental bacteria Comamonas testosteroni strains. BMC genomics 16:110. 
Liu WS, Ren PF, He SX, Xu L, Yang YL, Gu ZM, and Zhou ZG. 2013. Comparison of adhesive gut bacteria composition, immunity, and disease resistance in juvenile hybrid tilapia fed two different Lactobacillus strains. Fish \& Shellfish Immunology 35:54-62.

Louis P, Hold GL, and Flint HJ. 2014. The gut microbiota, bacterial metabolites and colorectal cancer. Nat Rev Microbiol 12:661-672.

Ma Y, Song L, Lei Y, Jia P, Lu C, Wu J, Xi C, Strauss PR, and Pei D-S. 2018. Sex dependent effects of silver nanoparticles on the zebrafish gut microbiota. Environmental Science-Nano 5:740-751.

Miller RA, and Harbottle H. 2018. Antimicrobial Drug Resistance in Fish Pathogens. Microbiol Spectr 6.

Monette MY, and McCormick SD. 2008. Impacts of short-term acid and aluminum exposure on Atlantic salmon (Salmo salar) physiology: a direct comparison of parr and smolts. Aquat Toxicol 86:216-226.

Nematollahi A, Decostere A, Pasmans F, and Haesebrouck F. 2003. Flavobacterium psychrophilum infections in salmonid fish. Journal of Fish Diseases 26:563-574.

Ni JJ, Yan QY, Yu YH, and Zhang TL. 2014. Factors influencing the grass carp gut microbiome and its effect on metabolism. Fems Microbiology Ecology 87:704-714.

Nikoskelainen S, Salminen S, Bylund G, and Ouwehand AC. 2001. Characterization of the properties of human- and dairy-derived probiotics for prevention of infectious diseases in fish. Appl Environ Microbiol 67:2430-2435.

Nowak A, and Libudzisz Z. 2006. Influence of phenol, p-cresol and indole on growth and survival of intestinal lactic acid bacteria. Anaerobe 12:80-84.

Park E-J, Sim J, Kim Y, Han BS, Yoon C, Lee S, Cho M-H, Lee B-S, and Kim J-H. 2015. A 13-week repeated-dose oral toxicity and bioaccumulation of aluminum oxide nanoparticles in mice. Archives of Toxicology 89:371-379.

Pascual J, Macian MC, Arahal DR, Garay E, and Pujalte MJ. 2009. Description of Enterovibrio nigricans sp nov., reclassification of Vibrio calviensis as Enterovibrio calviensis comb. nov and emended description of the genus Enterovibrio Thompson et al. 2002. International Journal of Systematic and Evolutionary Microbiology 59:698-704.

Perez T, Balcazar JL, Ruiz-Zarzuela I, Halaihel N, Vendrell D, de Blas I, and Muzquiz JL. 2010. Hostmicrobiota interactions within the fish intestinal ecosystem. Mucosal Immunology 3:355-360.

Pirarat N, Kobayashi T, Katagiri T, Maita M, and Endo M. 2006. Protective effects and mechanisms of a probiotic bacterium Lactobacillus rhamnosus against experimental Edwardsiella tarda infection in tilapia (Oreochromis niloticus). Vet Immunol Immunopathol 113:339-347.

Pirarat N, Pinpimai K, Rodkhum C, Chansue N, Ooi EL, Katagiri T, and Maita M. 2015. Viability and morphological evaluation of alginate-encapsulated Lactobacillus rhamnosus GG under simulated tilapia gastrointestinal conditions and its effect on growth performance, intestinal morphology and protection against Streptococcus agalactiae. Animal Feed Science and Technology 207:93-103. 
Qin C, Gong L, Zhang X, Wang Y, Wang Y, Wang B, Li Y, and Li W. 2018. Effect of Saccharomyces boulardii and Bacillus subtilis B10 on gut microbiota modulation in broilers. Anim Nutr 4:358-366.

Ran C, Huang L, Liu Z, Xu L, Yang Y, Tacon P, Auclair E, and Zhou Z. 2015. A Comparison of the Beneficial Effects of Live and Heat-Inactivated Baker's Yeast on Nile Tilapia: Suggestions on the Role and Function of the Secretory Metabolites Released from the Yeast. Plos One 10:e0145448.

Ridha MT, and Azad IS. 2012. Preliminary evaluation of growth performance and immune response of Nile tilapia Oreochromis niloticus supplemented with two putative probiotic bacteria. Aquaculture Research 43:843-852.

Ridha MT, and Azad IS. 2016. Effect of autochthonous and commercial probiotic bacteria on growth, persistence, immunity and disease resistance in juvenile and adult Nile tilapia Oreochromis niloticus. Aquaculture Research 47:2757-2767.

Russell SL, Gold MJ, Reynolds LA, Willing BP, Dimitriu P, Thorson L, Redpath SA, Perona-Wright G, Blanchet MR, Mohn WW, Finlay BB, and McNagny KM. 2015. Perinatal antibiotic-induced shifts in gut microbiota have differential effects on inflammatory lung diseases. Journal of Allergy and Clinical Immunology 135:100-U175.

Sihag RC, and Sharma P. 2012. Probiotics: the new ecofriendly alternative measures of disease control for sustainable aquaculture. Journal of Fisheries and Aquatic Science 7:72.

Standen BT, Peggs DL, Rawling MD, Foey A, Davies SJ, Santos GA, and Merrifield DL. 2016. Dietary administration of a commercial mixed-species probiotic improves growth performance and modulates the intestinal immunity of tilapia, Oreochromis niloticus. Fish \& Shellfish Immunology 49:427-435.

Standen BT, Rodiles A, Peggs DL, Davies SJ, Santos GA, and Merrifield DL. 2015. Modulation of the intestinal microbiota and morphology of tilapia, Oreochromis niloticus, following the application of a multi-species probiotic. Applied Microbiology and Biotechnology 99:8403-8417.

Sullam KE, Essinger SD, Lozupone CA, O'Connor MP, Rosen GL, Knight R, Kilham SS, and Russell JA. 2012. Environmental and ecological factors that shape the gut bacterial communities of fish: a metaanalysis. Molecular Ecology 21:3363-3378.

Uronis JM, Arthur JC, Keku T, Fodor A, Carroll IM, Cruz ML, Appleyard CB, and Jobin C. 2011. Gut microbial diversity is reduced by the probiotic VSL\#3 and correlates with decreased TNBS-induced colitis. Inflammatory Bowel Diseases 17:289-297.

Vignal C, Desreumaux P, and Body-Malapel M. 2016. Gut: An underestimated target organ for Aluminum. Morphologie 100:75-84.

Wang D, He Y, Liang J, Liu P, and Zhuang P. 2013. Distribution and source analysis of aluminum in rivers near Xi'an City, China. Environmental monitoring and assessment 185:1041-1053.

Wang MZ, Guozhong; Han, Junyan; Zhao, Jianxin; Zhang, Hao; Chen, Wei. 2018. Isolation of foodborne Lactic Acid Bacteria and detection of the colonization ability in mouse intestinal tract. $J$ Chin Inst Food Sci Tech 18:239-245. 
456

457

458

459

460

461

462

463

464

465

466

467

468

469

470

471

472

473

474

475

476

477

478

Wu F, Tang K, Yuan M, Shi X, Shakeela Q, and Zhang X-H. 2015. Studies on bacterial pathogens isolated from diseased torafugu (Takifugu rubripes) cultured in marine industrial recirculation aquaculture system in Shandong Province, China. Aquaculture Research 46:736-744.

Wu GF, Xiao XP, Feng PY, Xie FQ, Yu ZS, Yuan WZ, Liu P, and Li XK. 2017. Gut remediation: a potential approach to reducing chromium accumulation using Lactobacillus plantarum TW1-1. Scientific Reports 7.

Wu SG, Tian JY, Gatesoupe FJ, Li WX, Zou H, Yang BJ, and Wang GT. 2013. Intestinal microbiota of gibel carp (Carassius auratus gibelio) and its origin as revealed by 454 pyrosequencing. World Journal of Microbiology \& Biotechnology 29:1585-1595.

Yu L, Zhai Q, Liu X, Wang G, Zhang Q, Zhao J, Narbad A, Zhang H, Tian F, and Chen W. 2016. Lactobacillus plantarum CCFM639 alleviates aluminium toxicity. Appl Microbiol Biotechnol 100:1891-1900.

Yu LL, Zhai QX, Zhu JM, Zhang CC, Li TQ, Liu XM, Zhao JX, Zhang H, Tian FW, and Chen W. 2017. Dietary Lactobacillus plantarum supplementation enhances growth performance and alleviates aluminum toxicity in tilapia. Ecotoxicology and Environmental Safety 143:307-314.

Zhai Q, Li T, Yu L, Xiao Y, Feng S, Wu J, Zhao J, Zhang H, and Chen W. 2017. Effects of subchronic oral toxic metal exposure on the intestinal microbiota of mice. Science Bulletin 62:831-840.

Zhai Q, Yu L, Li T, Zhu J, Zhang C, Zhao J, Zhang H, and Chen W. 2016. Effect of dietary probiotic supplementation on intestinal microbiota and physiological conditions of Nile tilapia (Oreochromis niloticus) under waterborne cadmium exposure. Antonie Van Leeuwenhoek.

Zheng Y, Qu J, Qiu L, Fan L, Meng S, Song C, Bing X, and Chen J. 2016. Effect of 17alphamethyltestosterone (MT) on oxidation stress in the liver of juvenile GIFT tilapia, Oreochromis niloticus. Springerplus 5:338. 
Figure 1

Figure 1 Alpha diversity results for the gut microbiota of Nile tilapia

The data shown are the means \pm SEM for each group. Asterisks represent significant differences compared to the control group, $\mathrm{P}=0.007$ and 0.042 for Chao (A), $\mathrm{P}=0.006$ and 0.041 for Shannon (B).

A

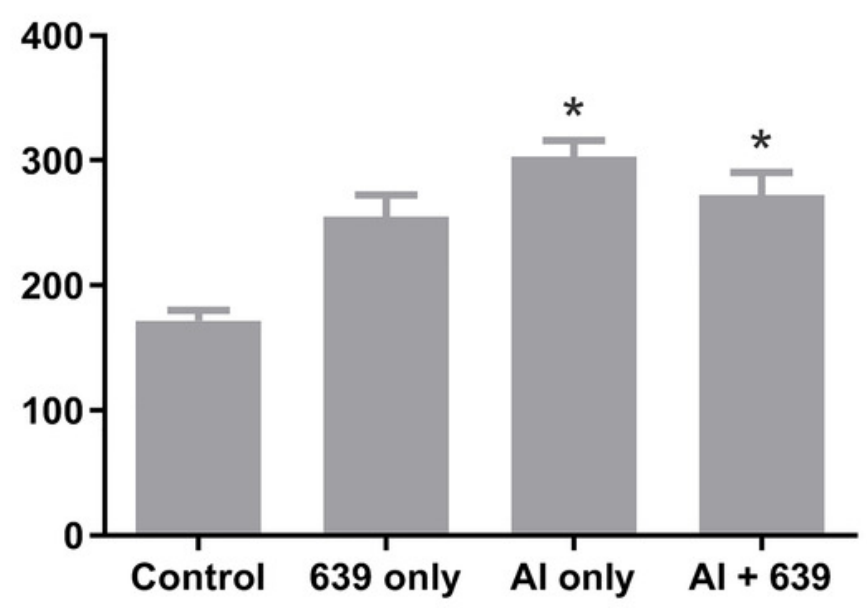

B

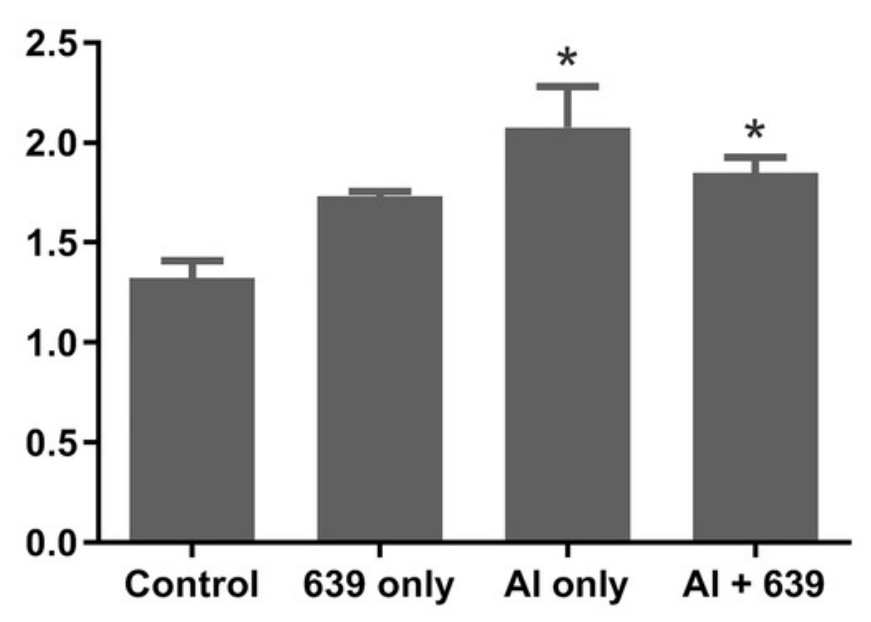


Figure 2

Principal coordinate score plots for the gut microbiota of Nile tilapia

(A) Unweighted unifrac distance. (B) PC1 values. The asterisk indicates the statistically significant differences $(P<0.05)$ between different groups, ns indicates no statistically significant differences.

A

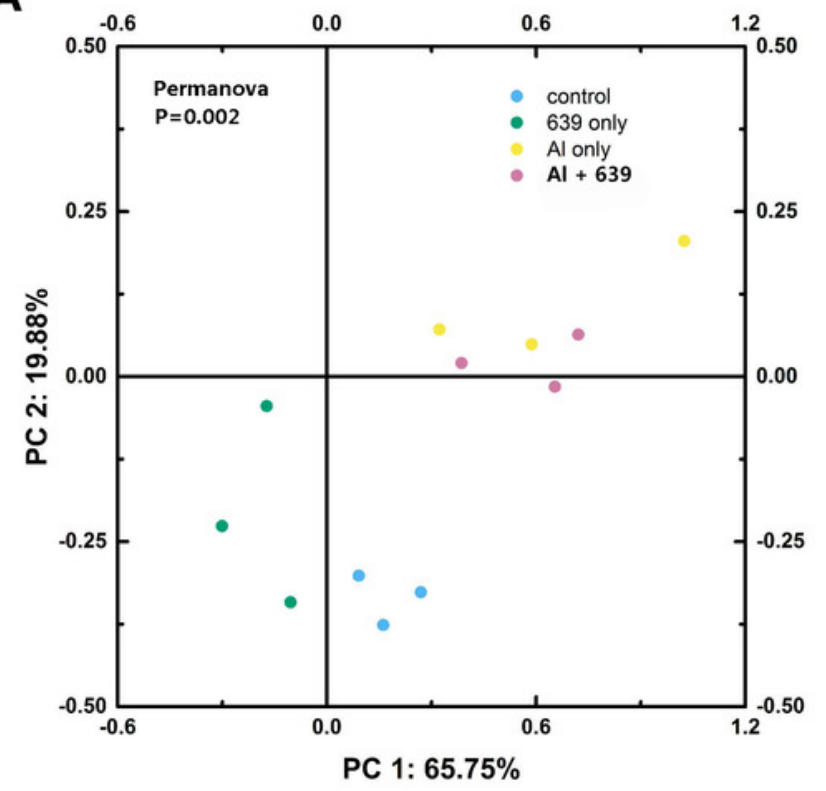

B 审 Control 追 639 Only 白 Al Only 审 Al+639

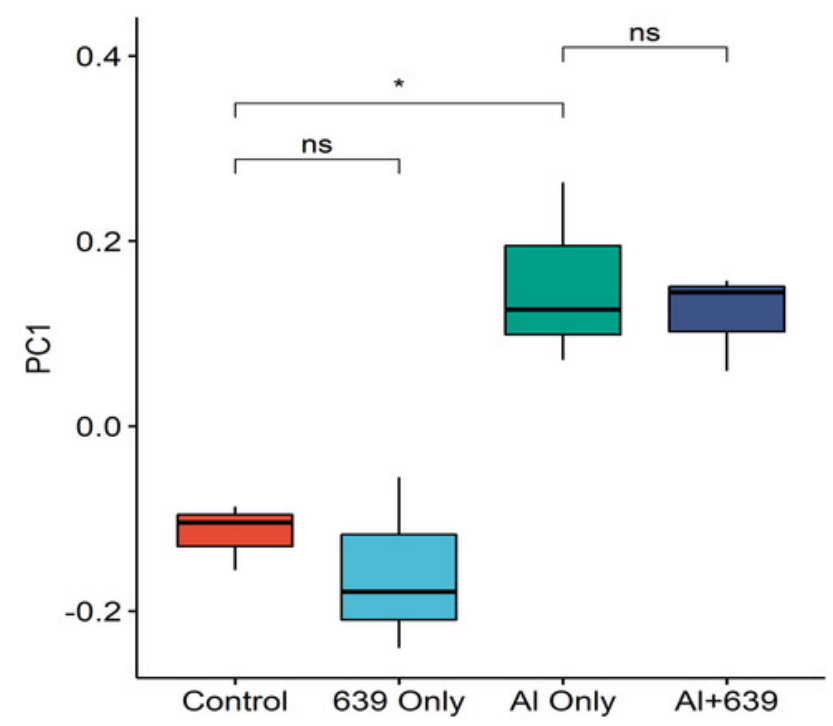


Figure 3

Effect of L. plantarum CCFM639 on the relative abundance (relative OTU composition) of the components of gut microbiota in Nile tilapia at the phylum level

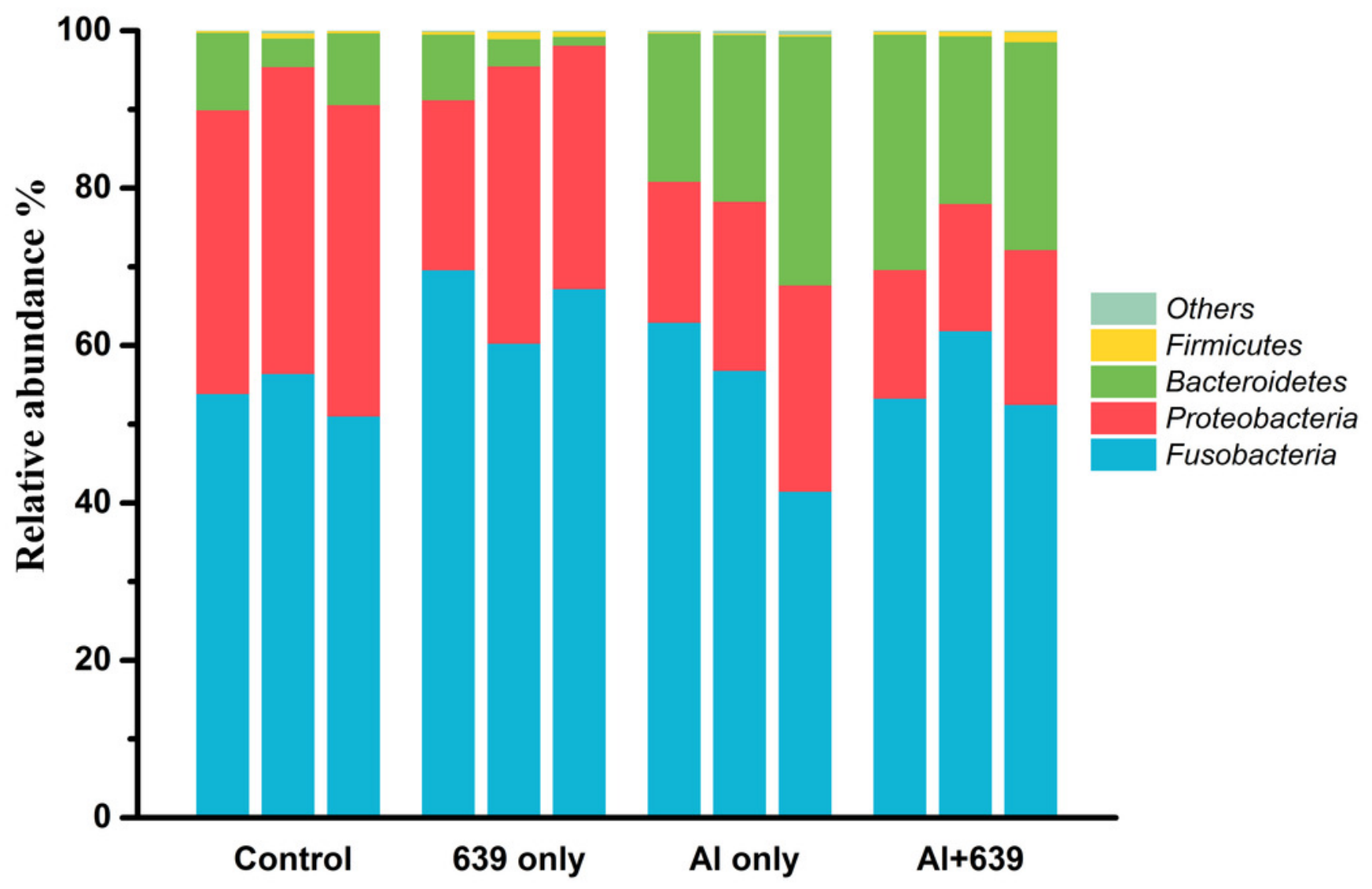




\section{Figure 4}

Effects of L. plantarum CCFM639 on the relative abundance (relative OTU composition) of the gut microbiota in Nile tilapia at the genus level

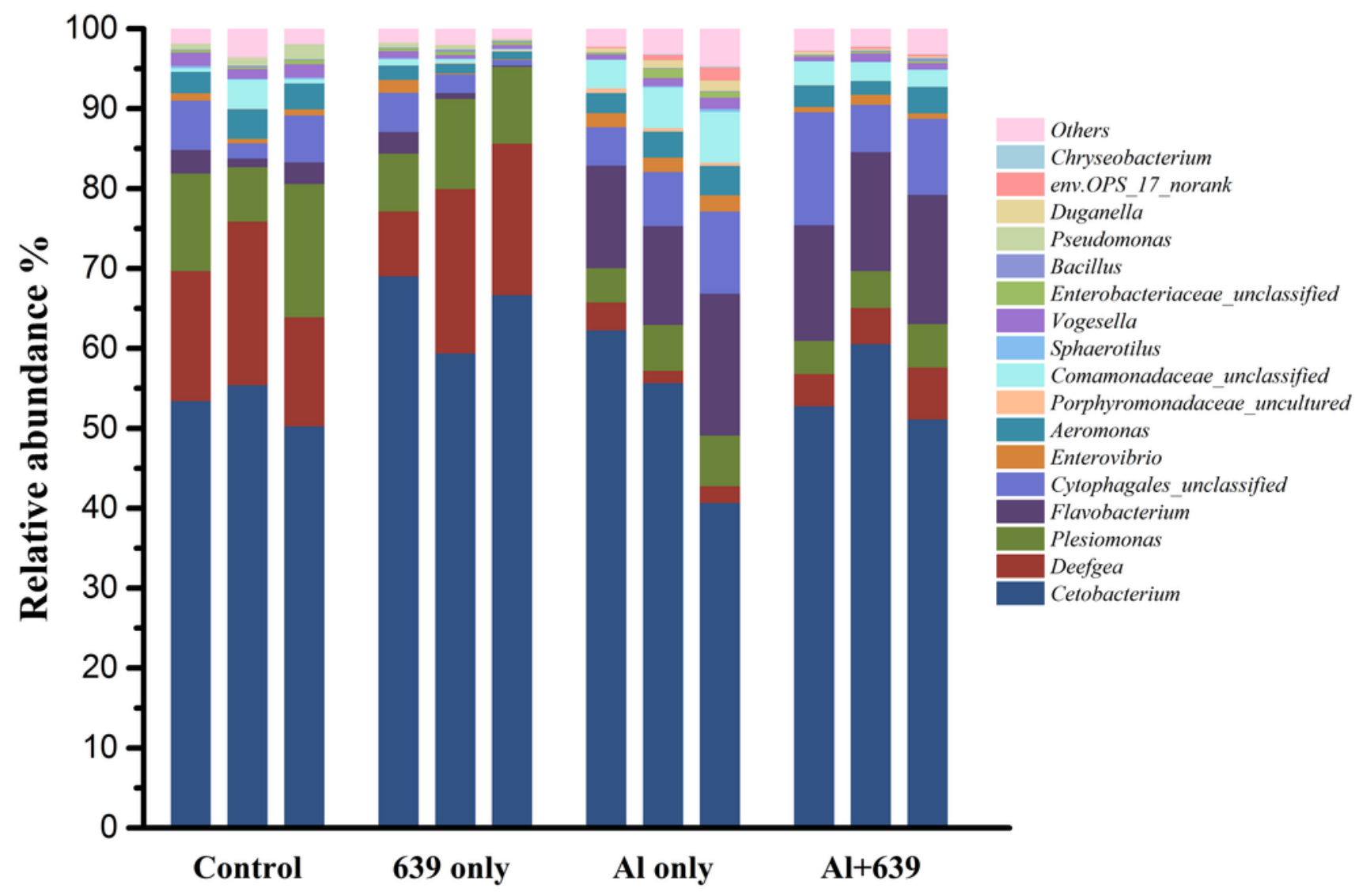


Figure 5

Relative abundance (relative OTU composition, $\% \pm$ SEM) of the gut microbiota in Nile tilapia at the genus level

Relative abundance of Cetobacterium, Deefgea, Plesiomonas and Flavobacterium; (B) Relative abundance of Cytophagales, Enterovibrio, Aeromonas and Porphyromonadaceae; (C) Relative abundance of Comamonadaceae, Sphaerotilus, Vogesella and Enterobacteriaceae; (D) Relative abundance of Bacillus, Pseudomonas, Duganella, env.OPS_17-norank and Chryseobacterium[i]. Data are expressed as mean \pm SEM.

A

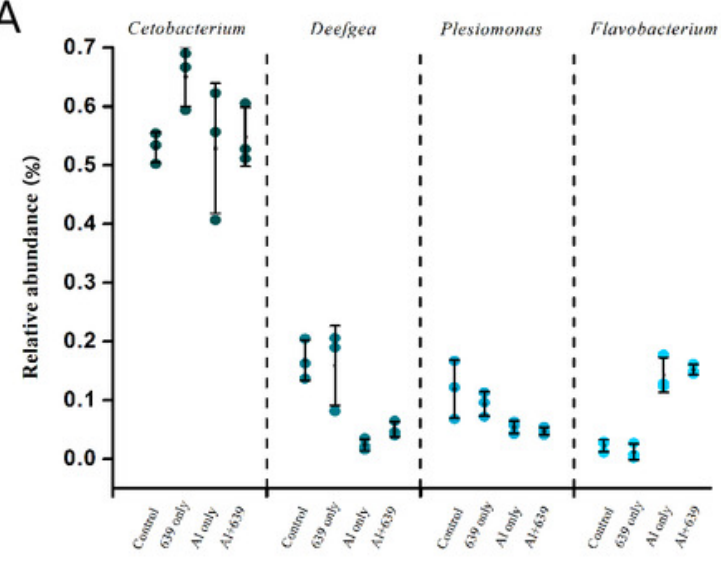

C

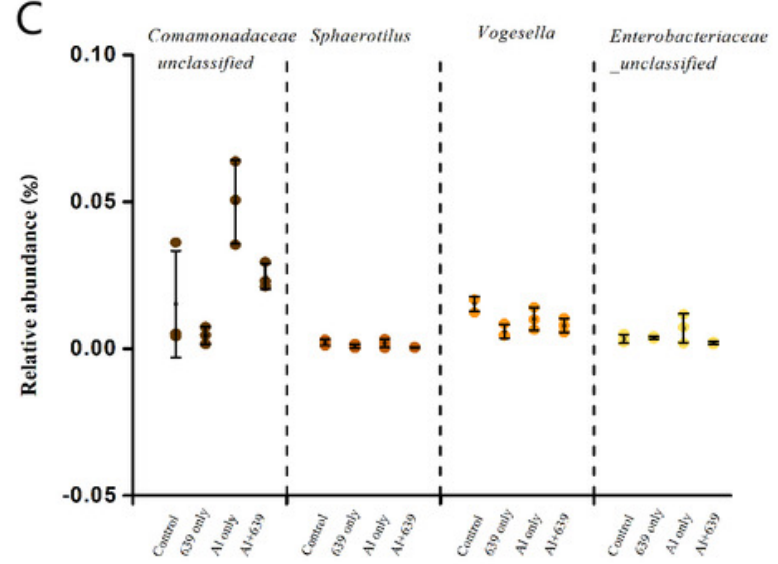

B

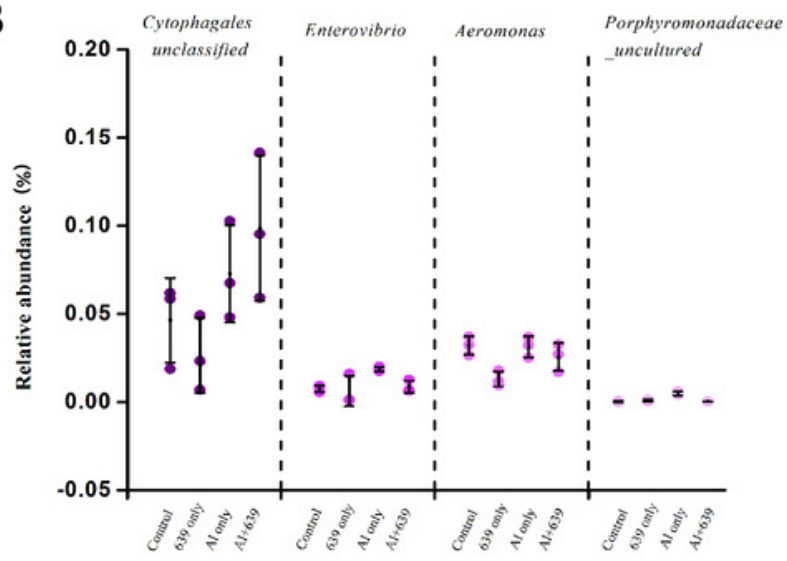

D

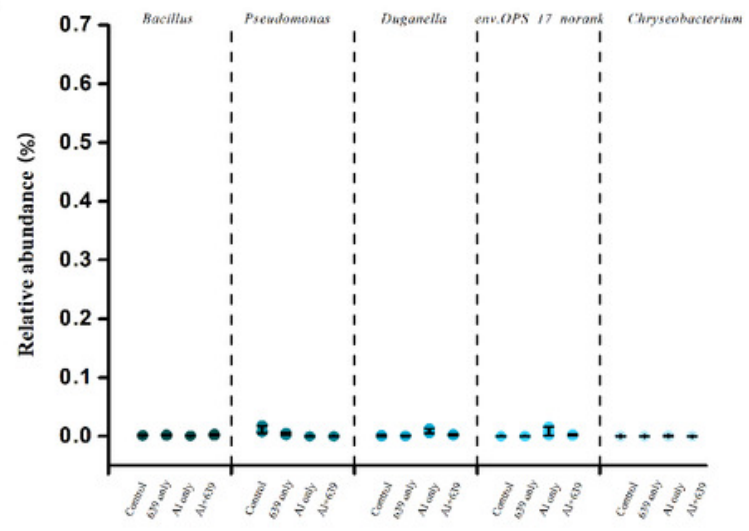


Figure 6

Potential protective mechanism of CCFM639 against aluminum induced gut injuries in Nile tilapia

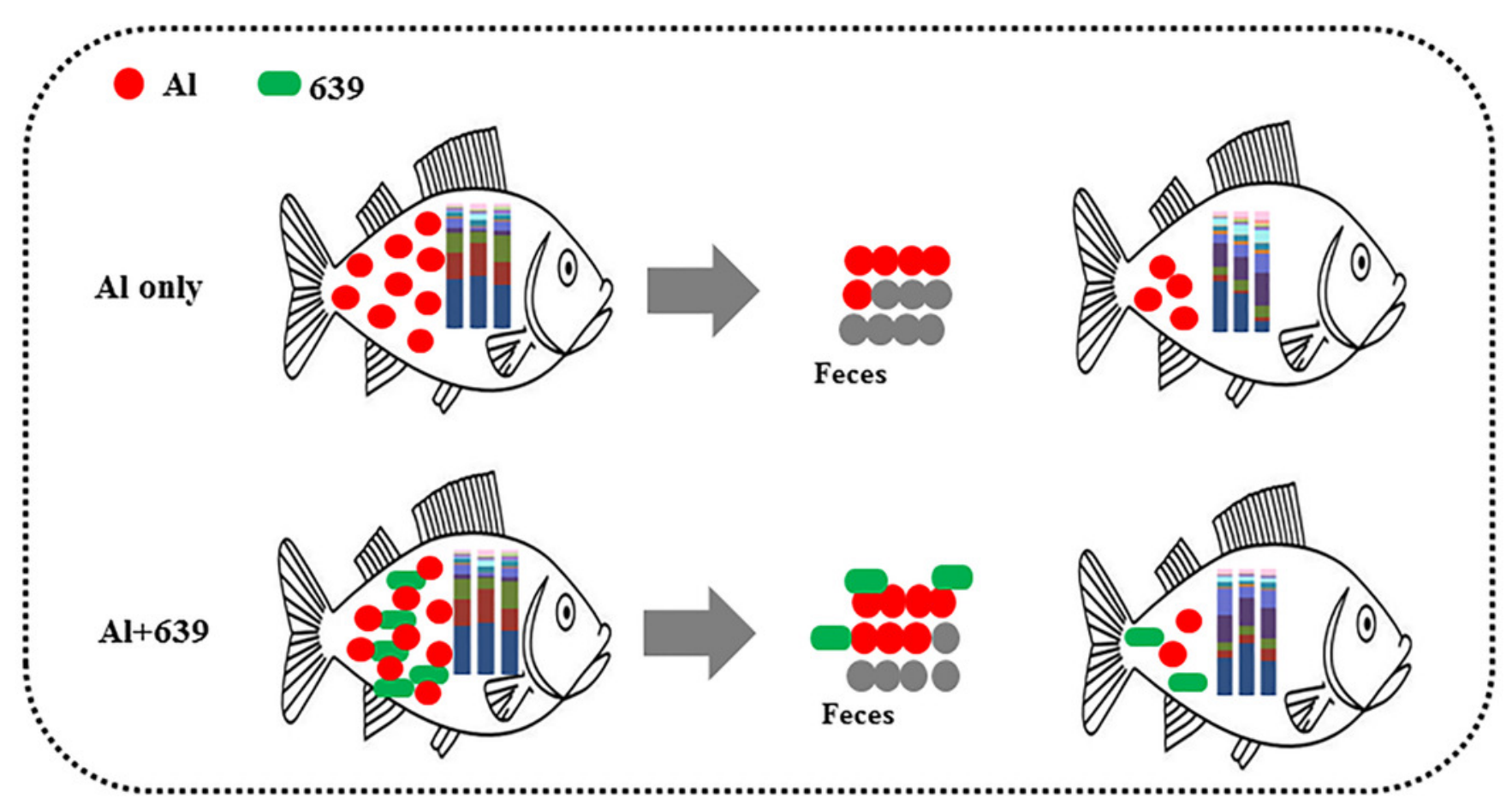




\section{Table 1 (on next page)}

Experimental groups for tilapia with and without Al exposure and CCFM639 feed 
1 Table 1. Experimental groups of tilapia with and without aluminum exposure and probiotic feed

\begin{tabular}{ll}
\hline Group & Experiment time (4 weeks) \\
\hline Control & Basic feed + normal water \\
639 only & probiotic feed + normal water \\
Al only & Basic feed + aluminum water \\
$\mathrm{Al}+639$ & probiotic feed + aluminum water \\
\hline
\end{tabular}

2 CCFM639 feed, feed containing L. plantarum CCFM639 at a concentration of $10^{8} \mathrm{CFU} / \mathrm{g}$;

3 aluminum water, an aqueous environment containing $2.73 \mathrm{mg} / \mathrm{L}$ of aluminum ions. 
Table 2 (on next page)

Effect of dietary supplementation with CCFM639 on Al contents in Nile tilapia feces 
1 Table 2. Effects of dietary supplementation with CCFM639 on aluminum contents in Nile 2 tilapia feces

\begin{tabular}{cccccc}
\hline \multirow{2}{*}{ Group } & \multicolumn{5}{c}{ Aluminum level $(\mathrm{mg} / \mathrm{kg})$} \\
\cline { 2 - 5 } & 0 week & Week 1 & Week 2 & Week 3 & Week 4 \\
\hline Control & $1.13 \pm 0.06^{\mathrm{aA}}$ & $1.54 \pm 0.07^{\mathrm{aA}}$ & $1.83 \pm 0.14^{\mathrm{aA}}$ & $1.80 \pm 0.10^{\mathrm{aA}}$ & $1.54 \pm 0.04^{\mathrm{aA}}$ \\
639 only & $1.19 \pm 0.04^{\mathrm{aA}}$ & $1.55 \pm 0.05^{\mathrm{aA}}$ & $1.68 \pm 0.13^{\mathrm{aA}}$ & $1.74 \pm 0.10^{\mathrm{aA}}$ & $1.58 \pm 0.06^{\mathrm{aA}}$ \\
Al only & $1.08 \pm 0.13^{\mathrm{aA}}$ & $22.33 \pm 1.31^{\mathrm{bB}}$ & $23.67 \pm 1.54^{\mathrm{bB}}$ & $25.22 \pm 1.32^{\mathrm{bB}}$ & $25.67 \pm 1.01^{\mathrm{bB}}$ \\
Al +639 & $1.05 \pm 0.08^{\mathrm{aA}}$ & $25.67 \pm 1.23^{\mathrm{cB}}$ & $33.14 \pm 2.53^{\mathrm{cC}}$ & $35.46 \pm 2.05^{\mathrm{cC}}$ & $33.79 \pm 2.37^{\mathrm{cC}}$ \\
\hline
\end{tabular}

3 The data shown are the mean \pm SEM for each group. The means with different superscript 4 lowercase letters differ significantly among groups, and the superscript capital letters indicate a 5 significant difference among time-points $(\mathrm{P}<0.05)$. 


\section{Table 3 (on next page)}

Effect of dietary supplementation with CCFM639 on L. plantarum quantification in Nile tilapia feces 
1 Table 3. Effects of dietary supplementation with CCFM639 on L. plantarum quantification in 2 Nile tilapia feces

\begin{tabular}{cccc}
\hline \multirow{2}{*}{ Group } & \multicolumn{3}{c}{ Number of L. plantarum $($ log copies/g feces $)$} \\
\cline { 2 - 4 } & Week 0 & Week 2 & Week 4 \\
\hline Control & $5.49 \pm 0.19^{\mathrm{aA}}$ & $5.47 \pm 0.09^{\mathrm{aA}}$ & $5.40 \pm 0.04^{\mathrm{aA}}$ \\
639 only & $5.52 \pm 0.01^{\mathrm{aA}}$ & $7.83 \pm 0.12^{\mathrm{bB}}$ & $7.73 \pm 0.02^{\mathrm{bB}}$ \\
Al only & $5.49 \pm 0.01^{\mathrm{aA}}$ & $5.24 \pm 0.08^{\mathrm{aB}}$ & $4.99 \pm 0.05^{\mathrm{cC}}$ \\
Al +639 & $5.54 \pm 0.13^{\mathrm{aA}}$ & $7.46 \pm 0.01^{\mathrm{cB}}$ & $7.31 \pm 0.13^{\mathrm{dB}}$ \\
\hline
\end{tabular}

3 The data shown are the mean \pm SEM for each group. The means with different superscript 4 lowercase letters differ significantly among groups, and the superscript capital letters indicate a 5 significant difference among time-points $(\mathrm{P}<0.05)$. 


\section{Table 4 (on next page)}

Effects of CCFM639 on aluminum-induced changes in relative abundance of Aeromonas, Enterovibrio, Comamonadaceae and Porphyromonadaceae of Nile tilapia 
Table 4. Effects of CCFM639 on aluminum-induced changes in relative abundance of Aeromonas, Enterovibrio, Comamonadaceae and Porphyromonadaceae of Nile tilapia

\begin{tabular}{ccccc}
\hline Group & Aeromonas & $\begin{array}{c}\text { Comamonad } \\
\text { aceae }\end{array}$ & Enterovibrio & Porphyromonadaceae \\
\hline Control & $3.20 \pm 0.31^{\mathrm{a}}$ & $1.52 \pm 1.05^{\mathrm{a}}$ & $0.76 \pm 0.10^{\mathrm{a}}$ & $0.03 \pm 0.02^{\mathrm{a}}$ \\
639 only & $1.31 \pm 0.25^{\mathrm{b}}$ & $0.45 \pm 0.17^{\mathrm{a}}$ & $0.62 \pm 0.50^{\mathrm{a}}$ & $0.09 \pm 0.03^{\mathrm{a}}$ \\
Al only & $3.14 \pm 0.34^{\mathrm{a}}$ & $5.00 \pm 0.82^{\mathrm{b}}$ & $1.86 \pm 0.08^{\mathrm{b}}$ & $0.49 \pm 0.06^{\mathrm{b}}$ \\
$\mathrm{Al}+639$ & $2.57 \pm 0.50^{\mathrm{a}}$ & $2.46 \pm 0.25^{\mathrm{a}}$ & $0.87 \pm 0.20^{\mathrm{a}}$ & $0.04 \pm 0.004^{\mathrm{a}}$
\end{tabular}

3 The data shown are the means \pm SEM for each group. The different superscript letters represent 4 significant differences between groups $(\mathrm{P}<0.05)$. 